\title{
DOS NOTAS «ARAGONESAS» (Y UNA VIEJA RESEÑA) EN TORNO A DON MANUEL ALVAR
}

\author{
José POLO \\ Universidad Autónoma de Madrid \\ jose.polo@uam.es
}

\section{Pasos no perdidos...}

Mi primer contacto, aunque solo fuese nominal (o sea, de mero aprendizaje de ficha) con la zona aragonesa en los estudios de don Manuel Alvar fue el libro, tópico en mi memoria, El dialecto aragonés (Biblioteca Románica Hispánica, III/Manuales, 7, Gredos, Madrid, 1953), obra que se convirtió pronto en verdaderamente clásica y, al menos para mí, también en mítica o de leyenda, porque, en los años en que yo comenzaba a formar mi biblioteca profesional, hacía tiempo que se encontraba agotada y nunca, que yo sepa, fue reimpresa, acerca de lo cual me preguntaba una y otra vez, pues no lograba entender que una obra tan instrumental, tan necesaria, quedara, en espacio temporal no dilatado, materialmente ausente. Años más tarde pude leer, dicho por el propio autor (véase más adelante 2-4, 2-5), la explicación -comprensible objetivamente, «técnicamente», pero no para un lector curioso y «avaricioso» en materia bibliográfica - de tal hecho. La verdad es que ahora, a gran distancia temporal, simplemente examinando el índice general de dicha obra, queda uno admirado del equilibrio metodológico y «factual» de dicho volumen, auténtico manual, con su muy completo aparato de índices auxiliares: de localidades citadas en el texto, de mapas, de materias, de palabras.

Avanzando en el tiempo, hacia 1974, se distribuyeron entre los que, arropados en la cátedra de Lengua Española de don Manuel Alvar, enseñábamos en la Universidad Complutense, se distribuyeron, decía, algunas obras para reseña y yo escogí (o me tocó: no recuerdo con precisión) Estudios sobre el dialecto aragonés, I (Institución «Fernando el Católico», Excma. Diputación Provincial, Zaragoza, 1973, 364 págs.), recensión —o, más bien, nota informativa- publicada en Archivo de Filologia Aragonesa, XVIII-XIX, 1967-1968 (aparecido, con gran retraso, en 1976), págs. 297-298 (ese volumen fue reimpreso, en la misma sede editorial, en 1987; véase la nota de José M. ${ }^{a}$ Enguita en la misma revista, XLII-XLIII, 1989, págs. 360-361). Junto a mi reseña aparecen las realizadas, para otras obras (una de ellas, del propio Alvar), por varios de los compañeros de tareas docentes en el mencionado centro universitario: la del llorado Julio Fernández-Sevilla y las de Mariano de Andrés Gutiérrez, 
María Jesús Fernández Leborans, José Antonio Mayoral y Vidal Alba de Diego. De otros estudios se ocupan las reseñas de Juan A. Frago Gracia, Ángeles Líbano Zumalacárregui y María Pilar Jaime Sisó, todos ellos de la Universidad de Zaragoza. Como uno de los rasgos, vamos a decir, de estilo de Manuel Alvar es enlazar, de un modo sencillo y sentido, lo «técnicamente filológico» con lo humano en general, me voy a permitir citar el último párrafo (pág. 10) de su «Presentación» del volumen:

Al final, un broche lírico de sencilla belleza [«Un zéjel aragonés del siglo xv»]. Y ahora, también, la voz que escuchamos es una voz aragonesa. Un viejo notario zaragozano —allá por 1416 - [,] harto de copiar protocolos[,] dejó que su pluma se explayara en unos versos emocionados. Ahora, también, tras tanto rigor y tanta aspereza, sea esta voz la llamada que nos haga volver a nuestra condición de humanistas.

En 1978, y editado por la misma institución del primer volumen, aparece, igualmente, Estudios sobre el dialecto aragonés, II. Con el mismo propósito ilustrativo del fondo siempre humano de don Manual Alvar, voy a reproducir, de su «Presentación», los dos últimos párrafos (pág. 10), que separo mediante pleca doble en negrita:

Son, pues, trabajos dispersos que reuno [reúno] ahora por su interés hacia un dialecto, [coma «errática» del original] que[,] por ser el mío, me inició en los trabajos lingüísticos. Poco es lo que he cambiado en ellos, salvo la [lo] estrictamente necesario (algún error, alguna vacilación, algún rasgo estilístico), o las repeticiones que pudieran aparecer en sitios diferentes: he procurado mantener las cosas como estaban. Si pertenecen a una etapa de mi quehacer científico, sería absurdo renegar de ella porque la crea superada. ¿Qué es lo que superamos? Cada yo actual es hijo de los yos [o yoes o yo-s] de ayer, y malo es renegar de lo que nos ha hecho ser. Mis trabajos cuentan por su voluntad y su fervor; si hoy me encuentro lejos de alguno, no puedo por menos que reconocer que sin ellos no sería quien soy. | | En 1941, cuando estudiaba en Zaragoza el primer curso de Letras, la Institución Fernando el Católico me acogió entre sus becarios. Con mucha vida cumplida, pero con idénticos entusiasmos que entonces, firmo este prólogo. Ahora, sin embargo, sé que la Comisión Permanente de la Institución «acordó por unanimidad» publicar este volumen. Mi gratitud no puede acallarse, por más que sean pobres las palabras para expresarlo. Lo eran entonces, 1941, cuando un mozo con muy pocos años fue recibido confiadamente lo son ahora - pobres, las palabras - cuando, 1975, el hombre maduro es recibido con cariño entrañable en la misma casa de la que salió; la casa a la que se vuelve - como al hogar paterno- cuando la vida se cansa de acongojar a la criatura.

\section{4}

Nos trasladamos ya al año 1998, en que, en la misma sede editorial de los dos primeros volúmenes, se publica el tercero, III, de la obra consabida, Estudios sobre el dialecto aragonés. En la «Presentación», titulada «¿Dialecto aragonés o geografia lingüística de Aragón?», se explica perfectamente la ruta científica que va de 1953, con su libro El dialecto aragonés, hasta el último momento de su investigación aragonesa. Esta presentación, que en realidad es más bien, desde el punto de vista técnico, una introducción, posee un valor historiográfico notable, pues aparece nítidamente, y con una proyección metodológica magistral, insuperable, lo que podríamos llamar la intrahistoria, trascendente en grado sumo, 
de su propia evolución al par del crecimiento de los problemas pendientes de resolución y de las amplias perspectivas que se iban abriendo conforme aumentaba el ordenado conocimiento de los hechos. Aunque cabría citar de ese espléndido texto multitud de pasajes esclarecedores, me basta con señalar la sostenida riqueza doctrinal y metodológica; pero no me resisto a citar los párrafos primero (sin rótulo, meramente introductorio; pág. 11) y último (titulado «Final»; págs. 23-24). De nuevo, me valgo de la pleca doble en negrita para señalar el final de la primera cita y el comienzo de la segunda:

Desde que en 1953 se publicó El dialecto aragonés [omito la nota 1, con los datos bibliográficos completos] hasta los días que corren, han pasado muchas cosas y la investigación sobre las hablas de nuestra región no podemos decir que se haya detenido. Factores de diverso tipo han activado el quehacer: por ejemplo, la creación de una especialidad de filología en la Universidad de Zaragoza (1967) y, consecuentemente, la provisión de unos cuadros de especialistas y el entusiasmo de los jóvenes estudiantes; la constancia de la Institución «Fernando el Católico» interesando el desarrollo de los estudios regionales; la valoración de las hablas vivas por los dialectólogos de la posguerra. Si aquel libro era una síntesis, treinta y cinco años más tarde debemos plantearnos qué significó y cómo debemos continuar. | He querido explicar un quehacer y el porqué de no hacer otros. Lo que un día era imperativo, otro dejó de serlo, y el imperativo se vio desde distinta perspectiva. Cada obra vale en función de su tiempo y no debemos sacarla de él para hacerle decir lo que ni pudo ni quiso. Pero tampoco hemos de creer en el valor de las repeticiones. Un método de trabajo [el de la geografía lingüística] ha acrecentado inmensamente nuestros conocimientos, y mal haríamos si no lo utilizáramos. Es posible que nos haga relegar al olvido lo que fue producto de entusiasmo y diligencias. Tampoco valen las lamentaciones. Si queremos hacer que la ciencia progrese, no debemos arregostarnos en las recachas invernizas.

Aparte de los anteriores, son algunos más los estudios de don Manuel Alvar relacionados con ese «gran complejo lingüístico» (el mundo del aragonés, de lo aragonés, de su articulación con realidades colindantes y de otros universos lingüísticos arropados por una ancha historia común). Aparte de en bibliografías en torno a su obra, aparecen señalados tales estudios en textos de solapa, etc., de los volúmenes mencionados en las presentes notas. Pero ahora quiero trasladarme al último trabajo que he leído (págs. 263-292) en esta zona, a saber, «Aragonés», capítulo suyo dentro de la obra, por él dirigida, Manual de dialectología hispánica. El español de España, Ariel, Barcelona, 1996. Insistiendo yo en la necesidad de respuesta a la pregunta que me hacía sobre la no reimpresión de su obra aragonesa de 1953, se nos vuelve a decir algo al respecto en el primer párrafo de dicho estudio (pág. 263):

En 1953 se publicó la primera obra de conjunto sobre El dialecto aragonés [omito la nota 1 , con los datos bibliográficos de esa obra más alguna otra referencia, a saber, a la revista Archivo de Filología Aragonesa, con su tomo LI en 1996...],. El que no haya sido sustituida por otra no dice sino que los materiales recogidos seguían siendo los universalmente válidos. Pero la publicación del atlas regional dio un sesgo totalmente diferente a buena parte de esos estudios [omito la nota, con los datos del atlas lingǘstico y etnográfico de Aragón, Navarra y la Rioja: 12 volúmenes; con la colaboración de Tomás Buesa, Antonio Llorente y Elena Alvar]. Porque entonces las hablas vivas cobraron una significación muy distinta de la que teníamos y vieron enriquecerse campos habitualmente bien estudiados 
(frontera catalano-aragonesa), otros (localidades de Zaragoza y Teruel) contaron una vida que les estaba negada.

\section{Microsistemas completos y obras completas}

En mi opinión, uno de los errores más graves que se pueden cometer en torno a la planificación científico-editorial de la obra de Manuel Alvar es intentar llevar a cabo directamente unas obras completas. La complejidad de la «estructura editorial» de lo publicado por este gran estudioso es verdaderamente magistral: vastedad de lo que se halla en letra de molde, cosas en prensa o pendientes de elaboración final, presencia de determinados trabajos en más de un volumen (por razones de agotamiento de alguno de ellos o por otras circunstancias, que ahora resultaría prolijo examinar) y otra serie de hechos fácilmente inteligibles para quien haya seguido mínimamente, con orden y concierto, la ilímite producción científica del maestro. Ahora bien, lo que es factible, aunque también resulte complejo, es ir asediando el terreno creando grandes piezas, con su imprescindible coherencia interna, proyectadas hacia unas obras completas a medio o largo plazo. Me refiero -es fácil adivinarlo- a lo que he denominado «microsistemas completos», que, en el caso de nuestro investigador, son bastante más que lo dado a entender por el «modesto» término MICROSISTEMA.

\section{2}

Si hiciéramos un recuento de las «regiones» o zonas científicas asediadas largamente por el maestro Alvar, dejando provisionalmente de lado la atención prestada, por ejemplo, a la delimitación de los conceptos de lengua, dialecto y afines, así como a la, hoy por hoy, inabarcable, incansable, labor de geografía y cartografía lingüísticas, me atrevería a afirmar que, probablemente, dos de los susodichos microsistemas más redondos $\mathrm{y}$, consecuentemente, a pesar de su extensión, menos alejados de una aprehensión global susceptible de convertirse en una planificación científico-editorial que desemboque en unas «obras completas de zona», dos de tales «regiones conceptuales», decía, podrían ser las de los estudios dedicados al español de Canarias y a lo aragonés (lengua, literatura, historia...). Como mero recordatorio de trabajos del primero de esos frentes, señalaré, por ejemplo, El español hablado en Tenerife (1959), Estudios canarios (I, 1968; II, 1993), Niveles socio-culturales en el habla de Las Palmas de Gran Canaria (1972), ediciones, que presento someramente, sin los datos bibliográficos completos, pero en las que personas como Ventura Doreste, Alfonso Armas, Maximiano Trapero y otros han aportado su granito o grano de arena cuidándolas, redactando los índices auxiliares o dirigiendo tal operación, etc. (véase, complementariamente, editado por Cristóbal Corrales y Dolores Corbella, Estudios de dialectología dedicados a Manuel Alvar, Universidad de La Laguna, 2000). Y, por lo que respecta, al «universo aragonés», ya señalé (véase atrás 1-5) la rica información sobre trabajos aragoneses del profesor Alvar que aparece en los textos de solapa de diversas obras a las que antes me he referido. Pero, además, en volúmenes como Leer para el recuerdo (Málaga, 1998), dentro del tomo I, en la sección CLARA Y BELLA ESPAÑA, aparecen textos como «Aragón, tierra bien poblada» (págs. 60-69; en el índice general se lee poblada; en su espacio textual, en cambio, probada) o «Flores de Aragón» (págs. 70-71); en otro volumen de recopilación, Silva de varia lección (Institución «Fernando el Católico», Zaragoza, 1992), la sección última (págs. 213-224) se titula precisamente ARAGÓN (véase, en forma paralela a lo señalado antes, el homenaje de 
Archivo de Filología Aragonesa, LIX-LX/2003-2004). En fin, cabría rastrear, ya en plan de búsqueda de todo lo que existe en la obra del doctor Alvar, alrededor de universos científicos determinados y, sin duda, la cosecha compensaría con creces el no poco esfuerzo realizado. Pero convendría llamar la atención sobre un peligro «editorial». Veámoslo...

\section{3}

Se trata de lo siguiente. Una de las frases de sabia presencia en la obra de Alvar, frase que vertebra una concepción honda de la ciencia y de la cultura de un conjunto de países, es esa tan exacta de variedad en la unidad y unidad en la diferenciación, quedánđome yo, con otro sintagma que me llevaría, si no estoy equivocado, a trabajos varios suyos, a saber (tal frase): unidad en la diversidad o, si se prefiere, ya de mi parte, unidad, siempre unidad, por encima de la diversidad (antes que a pesar de la diversidad, puesto que la diversidad es parte ínsita de la historicidad de los hechos del lenguaje, de cualquier entorno vital). ¿Qué quiere decir todo esto? Pues algo muy sencillo: incluso mi propuesta de mecanismos editoriales para «microsistemas completos» tiene sus peligros si no se adoptan determinadas medidas que frenen el peligro de «regionalizar desequilibradamente» en la obra de un autor, como Manuel Alvar, cuyo rasgo caracterizador esencial en sus investigaciones ha sido —así lo creo yo - su profunda hispanidad o, si se prefiere, más latamente, su «hispanicidad» ('el hecho/la intelección/la vivencia de lo hispánico'). Nos hallamos, pues en esa delicada zona de transición entre lo que posee una relativa autonomía científica y lo que, aunque se dé en determinados espacios geográficos, ha nacido, vive y se explica por la comprehensiva visión del conjunto (histórico). De todos modos, la observación atenta de la obra múltiple, generosa, de don Manuel Alvar contiene suficientes orientaciones como para integrar armónicamente las muchas piezas de un gigantesco mapa de la lengua española en el mundo. He hablado antes, a manera de ejemplo, de las zonas canaria y aragonesa, como, sin duda, podría haber extendido la ejemplificación a otros ámbitos. Aquí y allá contamos con estudiosos bien preparados, y con las naturales dosis de prudencia, como para que, si se llevan a cabo proyectos editoriales «regionales», en cuanto solución realista ante la imposibilidad técnica de unas obras completas directas, se hagan, decía, las cosas como a él le habría gustado, manteniendo íntegro su espíritu y, si es posible, mejorando incluso la letra: corrección de erratas, reajustes textuales de naturaleza varia, alguna vez rectificación de un dato, etc. Tanto si es ARAGón como si es CANARIAS - ambas en inmejorables condiciones de completitud textual para ello--, ese primer fruto de unas obras completas por zonas científicas (fabricando grandes piezas para su ulterior inserción en el mecanismo gigantesco del conjunto de la obra del maestro Alvar), quien presente ese primer microsistema cabal habrá dado las pautas para que otros continúen y entre muchos se logre algún día tener ante la vista la imponente obra de un estudioso con una vocación profunda y un magisterio dilatado, sin fronteras, estudioso que nos ha dejado, entre otras, la lección de una labor incansable, incesante, algo más que ejemplar.

\section{La anunciada «vieja reseña»}

En 1-2 ya apareció fichado el volumen Estudios sobre el dialecto aragonés, I (1973). Como recuerdo de esos tiempos vividos, y en homenaje a don Manuel Alvar, voy a reproducir aquí tal reseña, pero con esporádica intervención de ahora, entre corchetes, para rectificar 
alguna fecha o para completar algún dato; también he colocado en su orden original cuatro líneas de texto que habían aparecido trastocadas/trastrocadas: dislocadas. Sobra decir que se trata de una recensión hecha por alguien que más bien comenzaba su vida científica y sobre un libro de contenidos muy distantes de lo que era y es la base de su formación filológica. No obstante, vista muchos años después, creo que mantiene el nivel de una digna «nota informativa». Releamos, pues, ese escrito no tanto pensando en su autor, sino en el hecho de que gira en torno a una importante obra de un maestro:

Tratándose de un estudioso tan conocido como Manuel Alvar, sobra cualquier tipo de presentación general al presente [a este] trabajo. Con todo, no dejaremos de mencionar tres obras que bien pudieran considerarse como el marco amplio más en relación con el libro de ahora; a saber: Dialectología española (CUADERNOS BIBLIOGRÁfICOS; CSIC, Madrid, 1962; próximamente, nueva edición [no la hubo]), Textos hispánicos dialectales [. Antologia histórica] (I-II, CSIC, Madrid, 1960) y Poesia española dialectal [. Estudio, selección y notas de Manuel Alvar] ([Ediciones] Alcalá, Madrid, 1969 [1965]. En los tres hay apartado para el aragonés, esto es, tal «dialecto» dentro del contexto de una «dialectología española». Ciñéndonos a continuación al terreno de esa lengua-dialecto, cabe mencionar como marco, también amplio, de la obra que estamos reseñando, libros como El habla del campo de Jaca (Universidad de Salamanca, 1948), Documentos de Jaca (1362-1502). Edición y estudios lingüisticos (Institución «Fernando el Católico», Zaragoza, 1960) y[] finalmente, Proyecto de un Atlas Lingüistico-Etnográfico [Lingüistico y Etnográfico] de Aragón (Institución «Fernando el Católico», Zaragoza, 1963), al igual que Cuestionario del Atlas Lingüistico y Etnográfico de Aragón (CSIC, Sevilla, 1962 [1963]). No se nos escapa que en las últimas cuatro obras se trata de metodologías distintas; pero, aun así, nos interesa señalarlas como contexto - no tan lato- del libro que nos ocupa, [sobra la coma] por lo que más tarde se dirá.

Pues bien: agotado hace mucho tiempo dicho trabajo, el autor se ha propuesto acometer en forma definitiva - al menos para los materiales de que dispone- el estudio del aragonés. Por una parte, queda lo relativo al atlas lingüístico-etnográfico, proyectado y ya en marcha (línea de trabajo de la que, naturalmente, no nos ocuparemos en esta reseña); por otra, tenemos la línea «filológica», de la que sí nos estamos ocupando indirectamente. Porque, en efecto, es en este segundo frente donde hay que situar la presente obra como el primer fruto de la necesaria recopilación de todos sus estudios sobre el aragonés, además del aporte de nuevos trabajos o de la ampliación o «reestructuración» de algunos de los viejos. En esta dirección aragonesa «integral» queremos mencionar, casi como hermano gemelo cronológicamente y en la intención «aragonesa», su obra (en prensa) Caracteres de la literatura aragonesa [anunciado con ese título en texto de solapa de la obra reseñada, apareció finalmente como Aragón. Literatura y ser histórico, Ediciones Pórtico, Zaragoza, 1976]. Así que, presentado el contexto amplio y estrecho de la obra fichada al comienzo, nos disponemos a dar breve noticia de la misma [a dar breve noticia de ella, de dicha obra].

Los trabajos que aparecen aquí agrupados — señala el autor- abarcan un largo periodo de tiempo. Por eso, también, su diversidad temática y metodológica. Están unidos, sin embargo, por una misma y sostenida voluntad de conocimiento. Conste, desde ahora, su pluralidad y su coherencia. Unos vieron la luz fragmentados por necesidades de impresión; otros, reducidos a lo que podría decirse en una obra de carácter sintético; algunos limitados a problemas muy concretos, sólo más tarde tuvieron cabal desarrollo. Su reunión en este 
volumen les devuelve uniformidad, los reduce o los amplía. Vistas así las cosas, pienso que pueden ser útiles los muchos materiales que he recogido, y elaborado con mayor o menor extensión, y que de otro modo quedarían dispersos en publicaciones no siempre de fácil acceso, o cuya consulta - entre otros temas misceláneos- dista de ser cómoda (pág. 9).

El libro está dividido en cuatro partes: I. EL LATÍN NOTARIAL (contiene los siguientes trabajos: Grafias navarro-aragonesas; Elementos romances en el latín notarial aragonés(1035-1134); La formación del apellido en los antiguos documentos aragoneses). - II. HISTORIA Y LINGÜÍSTICA («Colonización» franca en Aragón: onomástica, repoblación, historia (Los «Establimentz» de Jaca del siglo XIII)). - III. TEXTOS ALJAMLADOS (Interpretación de un texto oscense en aljamia hebrea; Apostillas lingüisticas al «Fecho de Buliquía》). - IV. LITERATURA Y DIALECTOLOGÍA (Rasgos dialectales en la «Disputa del alma y del cuerpo» (siglo XIV); Un zéjel aragonés del siglo V). LA OBRA ACABA CON LAS Abreviaturas bibliográficas y los Índices (de palabras y general). Tratándose de trabajos conocidos por los especialistas y dada la solvencia científica del autor, nos abstenemos de presentar una información más detallada del contenido del libro. Tampoco osamos adoptar actitud crítica alguna (o meramente descriptiva en el contexto más amplio del aragonés en conjunto). Nos limitaremos, pues, para acabar, a citar las palabras con las que Alvar inicia la presentación del libro (pág. 9):

Durante mucho tiempo dediqué mis trabajos al estudio de la dialectología aragonesa. La inevitable inquietud por investigar otros campos y el desarraigo de la región desde 1943 me llevaron cada vez más lejos de mis presupuestos iniciales. Sin embargo, cuanto he publicado no ha sido otra cosa que bosquejos de proyectos más ambiciosos. Hoy, cuando mi posición científica - y personal - ha cambiado mucho con respecto a lo que quise hacer, e incluso hice, y no veo fácil llevar a cabo muchas tareas comenzadas, pienso que puede ser útil una compilación como la que ahora presento.

Muy útil, sin duda: por el valor intrínseco de los trabajos agrupados y por la enorme ventaja de poder consultarlos fácilmente, «terapia» indispensable dentro de la grave incomunicación profesional que nos aqueja «por mor de las circunstancias». Esperemos que próximamente vean la luz otros volúmenes sobre el aragonés proyectados por el autor y vaya quedando asediado — relativamente, claro está- este campo de estudio'. 